\title{
Ferromagnetism in the Ferromagnetic Yttrium Iron Garnet Film/Ferromagnetic Intermetallic Compound Heterostructure
}

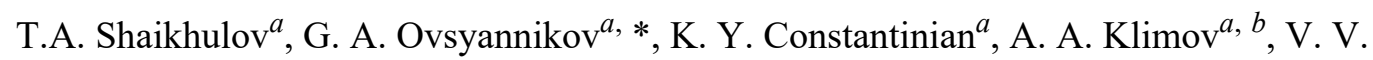

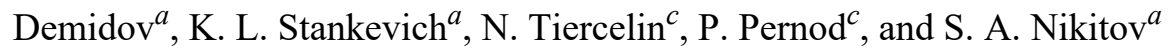 \\ a- Kotel'nikov Institute of Radio Engineering and Electronics, Russian Academy of Sciences, Moscow, 125009 Russia \\ b-Russian Technological University MIREA, Moscow, 119454 Russia \\ c- University of Lille, CNRS, Centrale Lille, ISEN, Univ. Valenciennes, UMR 8520 IEMN, Lille F-59000, France
}

\begin{abstract}
We report on the investigations of the magnetic properties and ferromagnetic resonance in the heterostructure consisting of an epitaxial yttrium iron garnet $\left(\mathrm{Y}_{3} \mathrm{Fe}_{5} \mathrm{O}_{12}\right)$ film and a nanometer rare-earth inter-metallic superlattice comprised of the $\left(\mathrm{TbCo}_{2} / \mathrm{FeCo}\right)_{n}$ exchange-coupled layers. The $(\mathrm{TbCo} / \mathrm{FeCo})_{n}$ super-lattice exhibits the giant magnetostriction and the controlled magnetic anisotropy induced by a magnetic field or elastic stresses. The magnetic interaction of the films in the $(\mathrm{TbCo} 2 / \mathrm{FeCo})_{n} / \mathrm{Y}_{3} \mathrm{Fe}_{5} \mathrm{O}_{12}$ heterostructure has been experimentally established and the spin current $\mathrm{f}$ lowing through their interface has been detected.
\end{abstract}

Keywords: ferromagnetic resonance, heterostructure, superlattice, magnetic anisotropy, spin current

\section{INTRODUCTION}

A structure with the spin current generation consists, as a rule, of two layers: a magnetic metal or a dielectric and a nonmagnetic metal. The spin current can be detected using the inverse spin-Hall effect (ISHE) in a material with a strong spin-orbit coupling by converting it into a conductive current $[1,2]$. How-ever, ISHE spin current detectors can be based not only on nonmagnetic metals. As was shown in [3-5], magnetic metals, e.g., permalloy $\mathrm{Ni}_{81} \mathrm{Fe}_{19}, \mathrm{Fe}, \mathrm{Co}$, and $\mathrm{Ni}$, can be used in spin current detectors. At the injection of the spin current into a ferromagnet, it is con-verted to the charge current due to the ISHE. It was shown that permalloy is characterized by the self-induced ISHE [6, 7].

The $\left(\mathrm{TbCo}_{2} / \mathrm{FeCo}\right)_{n}$ (TCFC) superlattices containing terbium $\mathrm{Tb}$, an element with the strong spinorbit coupling (the atomic weight is $Z=159)$, have a high Curie temperature $\left(T_{\mathrm{CU}} \sim 420 \mathrm{~K}\right.$ ), giant magnetostriction, and controlled induced magnetic anisotropy $[8,9]$. These properties of the TCFC films can be used to create spintronics elements. The strong spin- orbit coupling in TCFC holds out a hope of that these films can be used to detect the spin current using the ISHE. Ferromagnetic insulators, e.g., yttrium iron garnet $\mathrm{Y}_{3} \mathrm{Fe}_{5} \mathrm{O}_{12}$ (YIG), has an advantage over con-ducting ferromagnets due to the low attenuation of spin excitations (the Hilbert parameter is $\alpha=6-7 \times 10^{-5}$ ) and the high Curie temperature $\left(T_{\mathrm{CU}}=560 \mathrm{~K}\right)$, which ensures the high-efficiency spin pumping at room temperature [10]. It was reported that the spin current arises at the contact between the YIG film and the ferromagnetic FeNi film in which the voltage was detected by the ISHE [3-5]. In our previous work [11], we reported the preliminary results of investigations of the magnetic interactions in the TCFC/YIG heterostructures and $\mathrm{TCFC} / \mathrm{La}_{0.7} \mathrm{Sr}_{0.3} \mathrm{MnO}_{3}$. We observed broadening of the ferromagnetic resonance linewidth in the TCFC/YIG heterostructures and estimated the spin conductivity of the interface. The ISHE-induced electric voltage was experimentally observed in the TCFC film under the ferromagnetic resonance conditions in YIG. In this study, we report on the examination of the ferromagnetic resonance and the ISHE in the TCFC/YIG heterostructures in wide frequency and temperature ranges. The energy of the exchange coupling of the YIG and TCFC films in the heterostructure was determined. Using the measured ferromagnetic resonance linewidth in the TCFC/YIG heterostructures, the spin conductivity of the interfaces was estimated. The ISHE-induced electric voltage in the TCFC film under the conditions of the ferromagnetic resonance in YIG was experimentally observed.

\section{FERROMAGNETISM OF THE $\left(\mathrm{TbCo}_{2} / \mathrm{FeCo}\right)_{n} / \mathrm{Y}_{3} \mathrm{Fe}_{5} \mathrm{O}_{12}$ HETEROSTRUCTURE}

The $10-\mu \mathrm{m}$-thick YIG epitaxial film was grown on both sides of the 500 - $\mu \mathrm{m}$-thick (111) $\mathrm{Gd}_{3} \mathrm{Ga}_{5} \mathrm{O}_{12}$ substrate by molecular epitaxy. The substrate with the YIG film was cut into several pieces $4 \times 5 \mathrm{~mm}^{2}$ in size. On the top of the YIG film on one side, the $\left(\mathrm{TbCo}_{2} / \mathrm{FeCo}\right)_{n}$ intermetallic compound superlattice with thicknesses of 10,20, and $40 \mathrm{~nm}$ was deposited by magnetron sputtering. The magnetic anisotropy was induced in the TCFC superlattice by an additional magnetic field applied during deposition [11, 12]. The sample without the deposited TCFC film was used to study the autonomous YIG films.

A study of the magnetic moment of the films using a vibrating sample magnetometer (Fig. 1) showed that the TCFC superlattice saturation field $H_{S} \sim 1500$ Oe significantly exceeds the field $H_{S} \sim 200$ Oe for the YIG film. The magnetizations of both films lie in the substrate plane. At the change in the external magnetic field direction, the magnetization anisotropy of the TCFC superlattice can be clearly observed. 
At the same time, the magnetization anisotropy in the YIG film was not detected. The magnetization is $920 \mathrm{G}$ for the TCFC superlattice and $160 \mathrm{G}$ for the YIG film.

\section{FERROMAGNETIC RESONANCE IN THE $\left(\mathrm{TbCo}_{2} / \mathrm{FeCo}_{n} / \mathrm{Y}_{3} \mathrm{Fe}_{5} \mathrm{O}_{12}\right.$ HETEROSTRUCTURE}

The ferromagnetic resonance (FMR) in the heterostructures was measured using either a stripline in the microwave radiation ref lection mode in the frequency range of $1-20 \mathrm{GHz}$ or a bulk single-mode microwave resonator at a frequency of $9 \mathrm{GHz}$. The substrate with the film was pressed against the stripline with a resistance of $50 \Omega$. The magnetic component of the microwave field was directed along the long substrate side. A dc magnetic field was applied in the substrate plane perpendicular to the $\mathrm{f}$ lowing transport current. During the measurements in the range of $9 \mathrm{GHz}$, the angle between the transport current and the magnetic field changed within $180^{\circ}$ (see Section 4). During the microwave measurements, the hetero-structure temperature varied between $77-300 \mathrm{~K}$. The recorded spectra only included FMR lines of the YIG layers, since their thickness was larger than that of the TCFC layer by three orders of magnitude.

In the magnetic field modulation mode, the reflectivity derivative $d P / d H$ was measured. Figure 2 shows the room-temperature FMR spectra (the $d P / d H(H)$ dependences) for the TCFC/YIG heterostructure at microwave field frequencies of $F=1.8,1.7,1.5$, and $1.2 \mathrm{GHz}$. In the roomtemperature FMR spectrum of the heterostructure, two Lorentzian lines corresponding to two spin subsystems on both sides of the GGG substrate dominate.

The interplay between the microwave field and the magnetization of the YIG film leads to the occurrence of the spin-wave resonances. Due to the low attenuation in the YIG film in the in-plane magnetic field, at $\mathrm{H}<H_{0}\left(H_{0}\right.$ is the FMR resonance field), magneto-static surface waves (MSSW) are excited, the resonances of which manifest themselves in the form of FMR peaks. It is not impossible that the $d P / d H(H)$ peaks arising after two dominant peaks are caused by the MSSW resonance. In strong magnetic fields $\left(H>H_{0}\right)$, resonances of magnetostatic backward volume waves (MSBVWs) can be excited [13-17], but, in our case, their resonances are no observed. Figure 3 shows the dependence of the resonance field on the FMR frequency. The solid line shows the curve determined by the Kittel formula

$$
(\omega / \gamma)^{2}=H_{0}\left(H_{0}+4 \pi M_{0}\right)
$$

where $\omega=2 \pi F$ is the circular FMR frequency and $M_{0}$ is the YIG magnetization in the heterostructure. The best agreement between the experiment and the calculation is observed for $M_{0} \approx 100 \mathrm{G}$. The difference between the YIG magnetization measured on a vibrating sample magnetometer (Fig. 1) and the FMR frequency dependence of the resonance field (Fig. 3) probably originates from the degradation of the YIG magnetic parameters during deposition of the TCFC film.

The spectra of the heterostructure with the 40-nm-thick TCFC film with an increase in the microwave field frequency to $9 \mathrm{GHz}$ are well described by two Lorentzian lines (Fig. 4a). We assume that one of the lines corresponds to pure YIG and the other, to the TCFC/YIG bilayer. Due to the difference between the YIG and TCFC films by three orders of magnitude and the strong FMR attenuation in TCFC [11], the FMR from TCFC cannot be seen against the back-ground of the YIG signal. From the FMR line broadening caused by the excitation of two spin current ferromagnets at the interface, we determined which line corresponds to pure YIG (a linewidth of $15 \mathrm{Oe}$ ) and the YIG/TCFC bilayer (a linewidth of $26 \mathrm{Oe}$ ). The broadening of the FMR linewidth for the TCFC/YIG heterostructure is caused by an increase in the attenuation due to the spin current $\mathrm{f}$ low through the hetero-structure interface. Note that the YIG film before TCFC deposition is described by a single Lorentzian line with a width of 9 Oe (Fig. $4 b)$.

Figure 5 shows temperature dependences of the resonance fields of two selected highest-intensity lines of the heterostructure. The observed shift in the resonance fields can be explained by the effect of the inter-layer interaction of two neighboring ferromagnetic layers: the TCFC superlattice and the YIG film. A similar effect was previously observed on the TCFC/LSMO heterostructures [18]. It was shown in [18] that, in this case, the YIG FMR is described by Eq. (1), but with the replacement of $H_{0}$ by $H_{0}+$ $H_{J}$, where $H_{J}=J /(M d), J$ is the interlayer exchange constant, $M$ is the magnetization, and $d$ is the thickness of the TCFC-coated YIG film. 
To establish the temperature dependence of the interaction constant $J$, we determined, first, the temperature dependence of the magnetization of pure YIG using Eq. (1) (squares in Fig. 5) and, then, the temperature dependence of $H_{J}$ from the difference $\left(H_{2}-H_{1}\right)$. The obtained temperature dependences of $J$ are presented in Fig. 6 . The negative values of the interlayer interaction energy suggest that the TCFC/YIG interface is characterized by the antiferromagnetic interlayer interaction.

\section{SPIN CURRENT IN THE TCFC/YIG HETEROSTRUCTURE}

TCFC is characterized by the strong spin-orbit coupling and low $\left(10^{-4}-10^{-5} \Omega . \mathrm{cm}\right)$ resistivity in the investigated temperature range. To detect the ISHE-induced spin current in the YIG/TCFC bilayer, contact pads were formed on the film surface of the TCFC heterostructure. The sample was placed in the central plane of a TE102 rectangular microwave resonator. The ac field was directed along the voltage measurement line. A dc field was rotated in the sample plane. An ac field was induced by a Gunn diode operating at a frequency of $9 \mathrm{GHz}$. To increase the sensitivity, the ac field was modulated at a frequency of $100 \mathrm{kHz}$. The signal of the FMR sample was measured under the same conditions.

We found a signal that is the sum of two components: the first one is caused by the detection of the FMR signal of the sample at the contacts and the other results from the ISHE, which converts the spin current into the electric current. To separate these two contributions, the voltage signals were measured in two oppositely directed external magnetic fields. The upper plot in Fig. 7a is the FMR spectrum recorded at the modulation of the external microwave field. Upon rotation of the field by $180^{\circ}$, the FMR signal does not change its sign, while the spin current signal changes its sign for the opposite (see, for example, [19]). Thus, the spin current component can be distinguished by subtracting the signals obtained in the oppositely directed magnetic fields. Figures $7 \mathrm{~b}$ and $7 \mathrm{c}$ show the signals measured at the contacts to the TCFC film. Figure 7d presents the result of subtraction of two curves, which yields a spin current signal. It can be seen that only one peak corresponds to the spin cur rent. This is explained by the fact that the spin current arises at the YIG/TCFC interface, while the second side does not affect the spin current. Since the FMR signal at the contacts is comparable with the spin cur- rent signal, even a minor error of the experimental setup distorts the extracted spin current signal, which complicates the quantitative study of the spin current characteristics.

Then, we obtained the angular dependences of the FMR signals and the voltages on the contacts at the opposite directions of the external magnetic field. For each angle, the spectra were approximated by two Lorentzian lines and the intensity of each signal was calculated. Figure 8a shows the angular dependence of the signal intensity (areas of the detected lines). The dc fields are directed oppositely (red and black dots). As was shown above, the difference between the intensities of the signals with the oppositely directed fields will characterize the spin current and the sum of the signals will characterize the FMR signal (see Fig. 8b). In the plots, the intensity is normalized to the maxi- mum FMR signal.

Using the data presented in Section 3, we can determine the spin conductivity of the TCFC/LSMO heterointerface. We assume that the broadening of the heterostructure line $\alpha=\alpha 0+\alpha^{\prime}$ only occurs due to the spin current flow through the YIG/TCFC boundary. In this case, the spin conductivity is [20]

$$
g_{\mathrm{eff}}^{\uparrow \downarrow}=\frac{4 \pi \gamma_{g} M_{s} d_{\mathrm{YIG}}}{g \mu_{\mathrm{B}} \omega_{f}}\left(\Delta H_{\mathrm{YIG} / \mathrm{TCFC}}-\Delta H_{\mathrm{YIG}}\right),
$$

where $\gamma=17.605 \times 10^{6} \mathrm{~s}^{-1} \mathrm{G}^{-1}$ is the gyromagnetic ratio for the electron, $\omega=2 \pi \times 9.51 \times 10^{9} \mathrm{~s}^{-1}$ is the angular frequency, $M_{S}=100$ Oe is the magnetization of the YIG film, $d$ YIG $=5 \mu \mathrm{m}$ is the YIG film thick-ness, $\mu \mathrm{B}=9.274 \times 10^{-21} \mathrm{erg} / \mathrm{G}$ is the Bohr magneton, and $g=2$ is the Landé factor. At room temperature, we obtained the FMR linewidth broadening after deposition of the TCFC film by $\Delta H \mathrm{YIG} / \mathrm{TCFC}-\Delta \mathrm{HYIG}=3$ Oe and, consequently, $g_{\mathrm{eff}}=3.1 \times 10^{20} \mathrm{~m}^{-2}$. For comparison, in $[21,22]$, the values of $g_{\text {eff }}=2.1 \times 10^{19} \mathrm{~m}^{-2}$ for the Py $/ \mathrm{Pt}$ interface and $g_{\text {eff }}=4.8 \times 10^{20} \mathrm{~m}^{-2}$ for the YIG/Pt interface were obtained. 


\section{CONCLUSIONS}

The experimental study of the magnetic properties of the heterostructures consisting of epitaxial YIG garnet coated with a rare-earth TCFC intermetallic superlattice showed that the magnetic interaction in the heterostructure is antiferromagnetic. The energy of the exchange coupling of the YIG and TCFC films in the heterostructure was determined. The observed broadening of the ferromagnetic resonance line was attributed to the spin current flow through the TCFC/YIG interface. Using the measured ferromagnetic resonance linewidths in the TCFC/YIG hetero- structures, the spin conductivity of the interfaces was estimated. An electrical voltage induced by the inverse spin Hall effect in the TCFC film under the ferromagnetic resonance conditions in YIG was experimentally observed.

\section{ACKNOWLEDGMENTS}

The authors are grateful to V.A. Atsarkin, A.M. Petrzhik, V.L. Preobrazhenskii, and A.V. Shadrin for useful discussion of the results and help in the measurements.

\section{FUNDING}

This study was carried out under the state assignment and supported in part by the Russian Foundation for Basic Research, projects no. 18-57-16001 and 19-07-00143 and the Joint International Laboratory LEMAC-LICS.

\section{REFERENCES}

1. M. I. Dyakonov and V. I. Perel, Phys. Lett. A 35, 459 (1971).

2. E. Saitoh, M. Ueda, H. Miyajima, and G. Tatara, Appl. Phys. Lett. 88, 182509 (2006).

3. B.F.MiaoS.Y.Huang,D.Qu,andC.L.Chien,Phys. Rev. Lett. 111, 066602 (2013).

4. P. Hyde, Lihui Bai, D. M. J. Kumar, B. W. Southern, C.-M. Hu, S. Y. Huang, B. F. Miao, and C. L. Chien, Phys. Rev. B 89, 180404(R) (2014).

5. F. Yang and P. C. Hammel, J. Phys. D 51, 253001 (2018).

6. Tsukahara, Y. Ando, Y. Kitamura, H. Emoto, E. Shi- koh, M. P. Delmo, T. Shinjo, and M. Shiraishi, Phys. Rev. B 89, 235317 (2014).

7. L. Chen, S. Ikeda, F. Matsukura, and H. Ohno, Appl. Phys. Express 7, 013002 (2014).

8. H.LeGall, J.Ben, F.Socha, N.Tiercelin, V.Preobrazhensky, and P. Pernod, J. Appl. Phys. 87, 5783 (2000).

9. E.Quandt,A.Ludwig,D.G.Lord,andC.A.Faunce, J. Appl. Phys. 83, 7267 (1998).

10. Y. Kajiwara K. Harii, S. Takahashi, J. Ohe, K. Uchida, M. Mizuguchi, H. Umezawa, H. Kawai, K. Ando, K.Takanashi, S. Maekawa, and E. Saitoh, Nature (London, U.K.) 464, 262 (2010).

11. G. A. Ovsyannikov, V. V. Demidov, T. A. Shaikhulov, A. A. Klimov, N. Tiercelin, P. Pernod, and S. A. Nikitov, Phys. Solid State 61, 1652 (2019).

12. A. S. Grishin, G. A. Ovsyannikov, A. Klimov, V. V. De midov, K. Y. Constantinian, I. V. Borisenko, V. L. Pre- obrazhensky, N. Tiercelin, and P. Pernod, J. Electron. Mater. 47, 1595 (2018).

13. C. W. Sandweg, Y. Kajiwara, K. Ando, E. Saitoh, and B. Hillebrands, Appl. Phys. Lett. 97, 252504 (2010).

14. B. A. Kalinikos and A. N. Slavin, J. Phys. C 19, 7013 (1986); A. A. Serga, A. V. Chumak, and B. Hillebrands, J. Phys. D 43, 264002 (2010).

15. J. Barak and U. Lachish, J. Appl. Phys. 65, 1652 (1989).

16. I. Laulicht, J. T. Suss, and J. Barak, J. Appl. Phys. 70, 2251 (1991).

17. S. Dushenko, Yu. Higuchi, Yu. Ando, T. Shinjo, and M. Shiraishi, Appl. Phys. Express 8, 103002 (2015).

18. V. V. Demidov and G. A. Ovsyannikov, J. Appl. Phys. 122, 013902 (2017).

19. A. Azevedo, L. H. Vilela-Leão, R. L. Rodríguez- Suárez, A. F. Lacerda Santos, and S. M. Rezende, Phys. Rev. B 83, 144402 (2011).

20. Y. Tserkovnyak, A. Brataas, G. E. W. Bauer, and B. I. Halperin, Rev. Mod. Phys. 77, 1375 (2005).

21. O. Mosendz, V. Vlaminck, J. E. Pearson, F. Y. Fradin, G. E. W. Bauer, S. D. Bader, and A. Hoffmann, Phys. Rev. 82, 214403 (2010).

22. M. Rezende, R. L. Rodriguez-Suarez, M. M. Soares, L. H. Vilela-Le, D. Ley Dominguez, and A. Azeved, Appl. Phys. Lett. 102, 012402 (2013). 


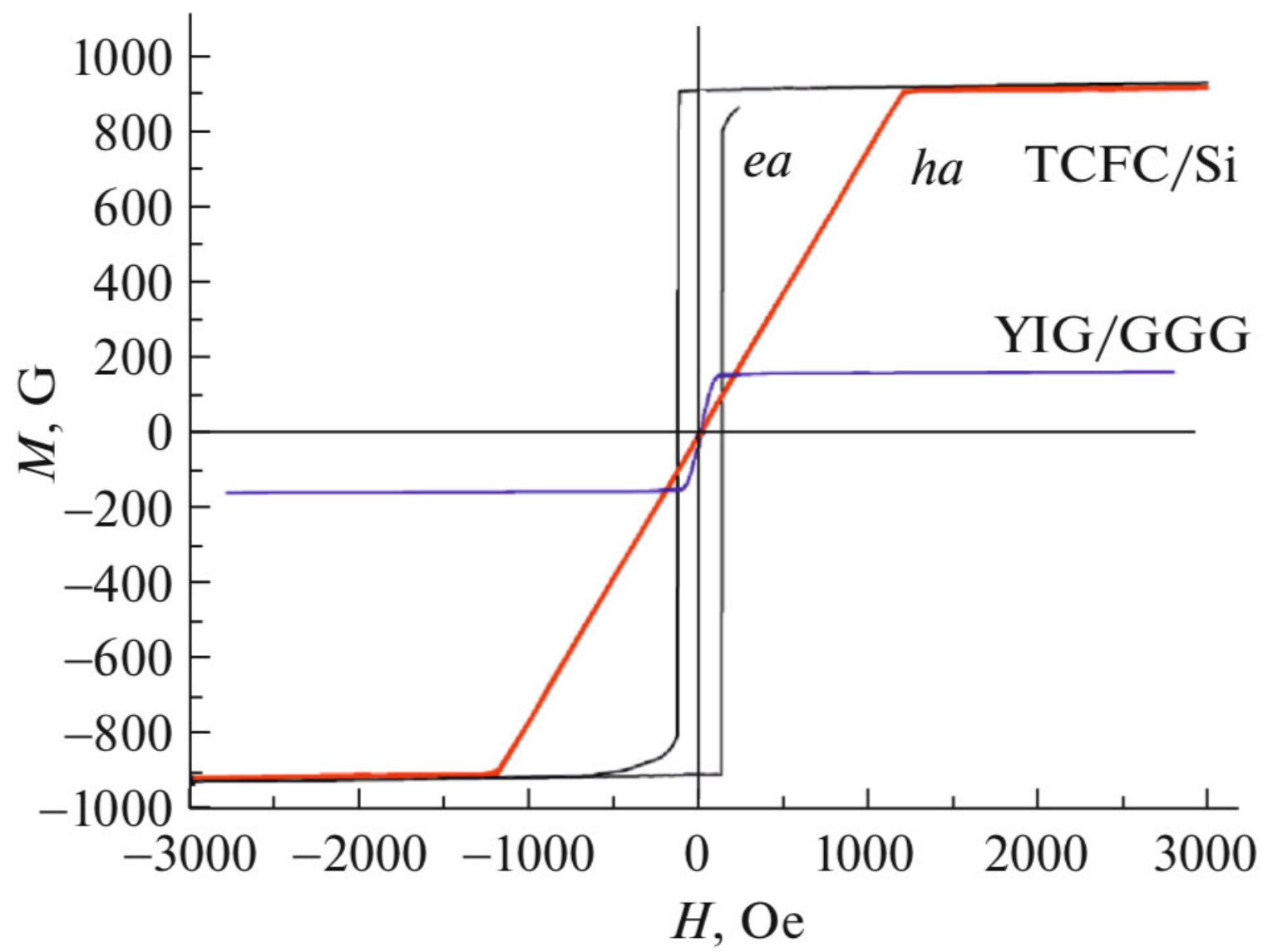

Fig. 1. Dependence of the magnetization of the YIG/GGG and TCFC/Si heterostructures on the external magnetic field directed along the easy $(e a)$ and hard $(h a)$ magnetization axes at $T=300 \mathrm{~K}$. 


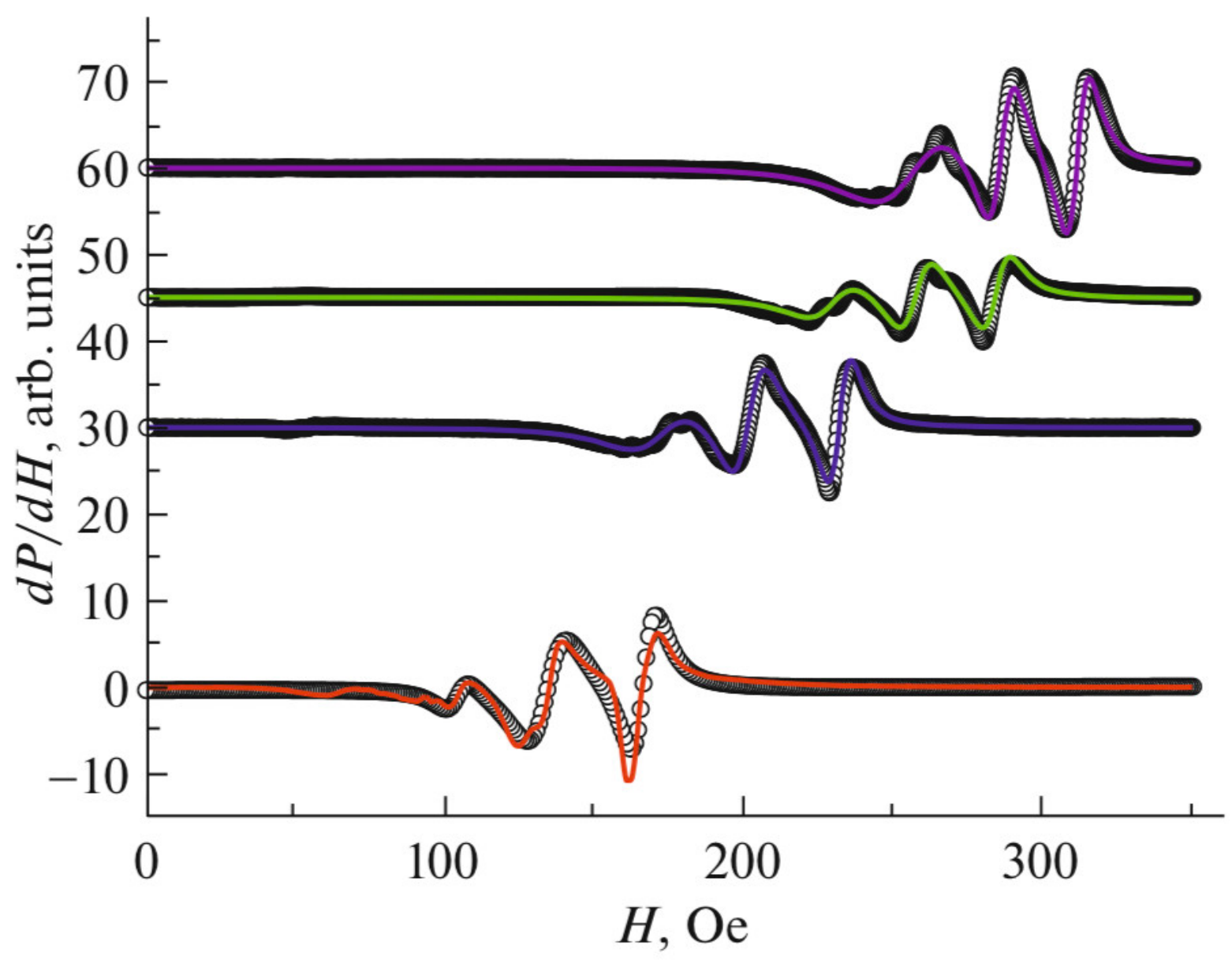

Fig. 2. FMR spectra $(d P / d H)$ of the TCFC/YIG hetero- structure at a TCFC film thickness of $10 \mathrm{~nm}$ and frequencies of $F$ $=1.8,1.7,1.5$, and $1.2 \mathrm{GHz}$ (top down) at a tem- perature of $T=300 \mathrm{~K}$. For clarity, the spectra are shifted along the ordinate axis. Points correspond to the experi- ment and solid lines, to the fitting of the curves by three Lorentzian lines. 


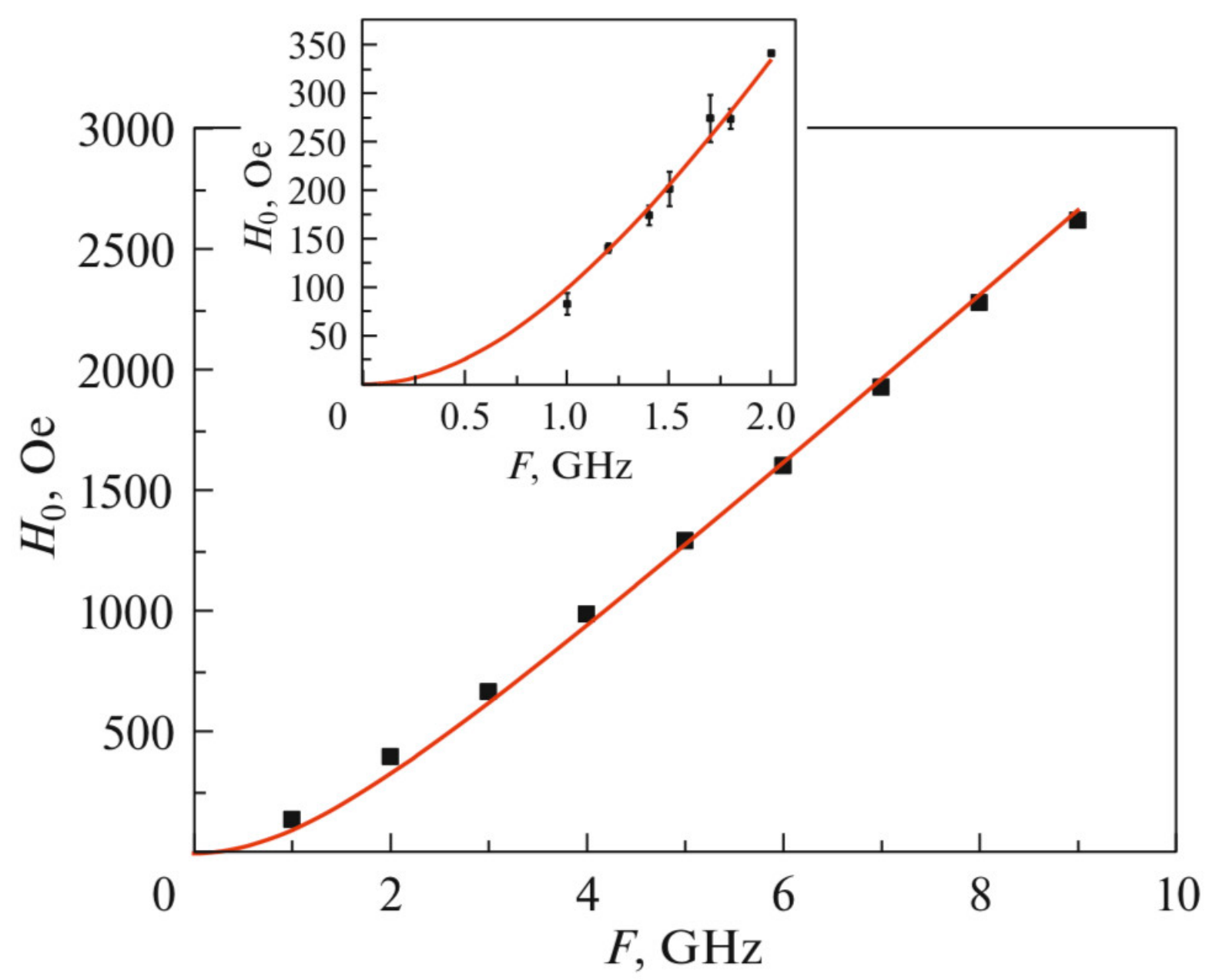

Fig. 3. Frequency dependence of resonance field $H_{0}$ for YIG in the TCFC/YIG heterostructure with a 40-nm- thick TCFC film. The solid line shows the dependence (1) for a magnetization of $M_{0} \approx 100 \mathrm{G}$. Inset: frequency dependence of $H_{0}$ in the low-frequency range. The error shown corresponds to the frequency dependence of the difference between the resonance fields of two lines with the highest intensities. 

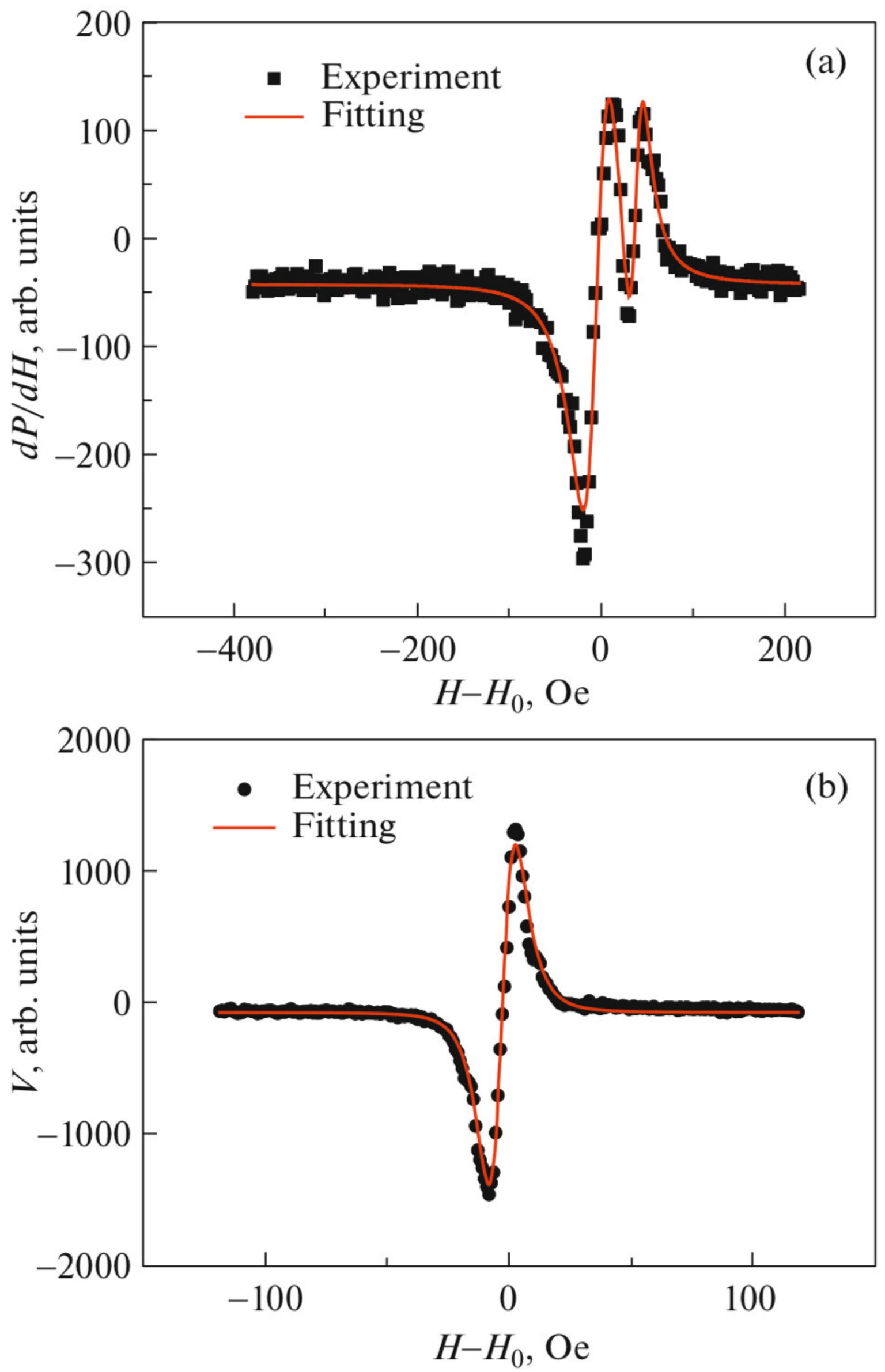

Fig. 4. (a) FMR spectra of the TCFC/YIG heterostructure with a 40-nm-thick TCFC film. (b) FMR spectrum of the YIG film before TCFC deposition. The microwave field frequency is $9 \mathrm{GHz}$. 


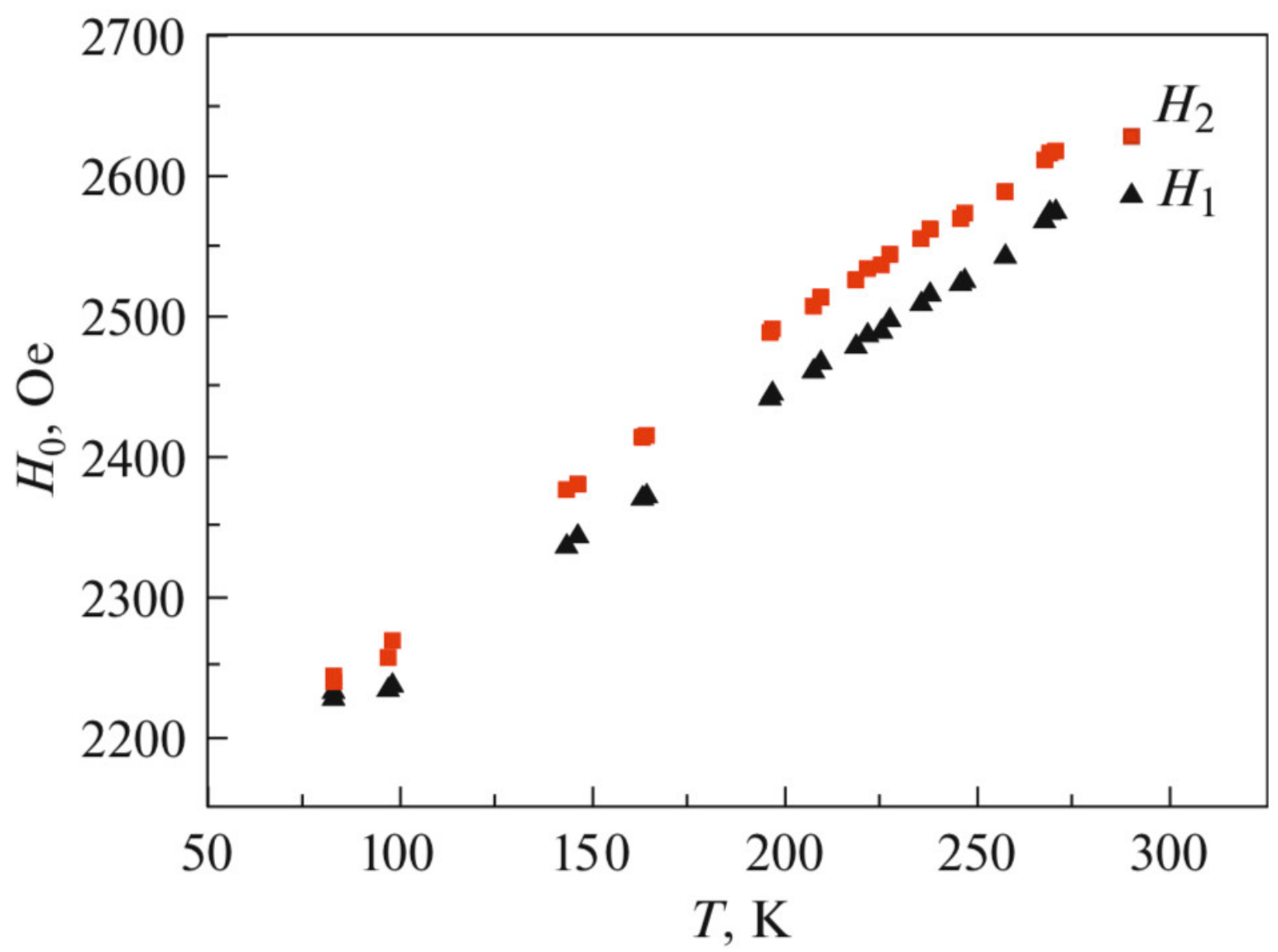

Fig. 5. Temperature dependences of resonance field $H_{0}$ for two peaks in the FMR spectrum of the TCFC/YIG heterostructure with a 40-nm-thick TCFC film. Triangles $\left(H_{1}\right)$ correspond to the YIG/TCFC bilayer and squares $\left(H_{2}\right)$, to YIG. The microwave frequency is $9 \mathrm{GHz}$. 


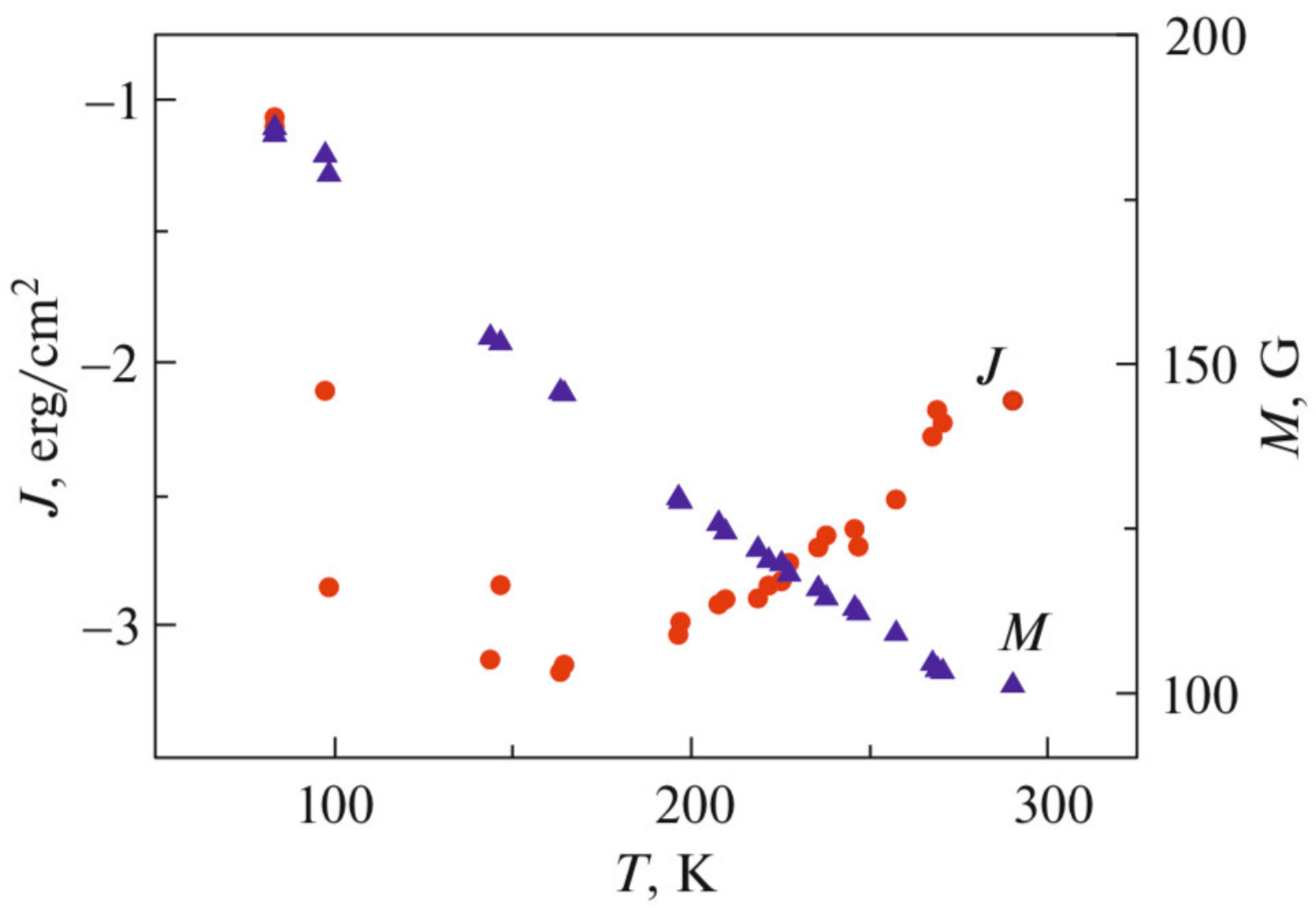

Fig. 6. Temperature dependences of the magnetization and exchange coupling energy in the TCFC/YIG heterostructure with a 40-nm-thick TCFC film. 


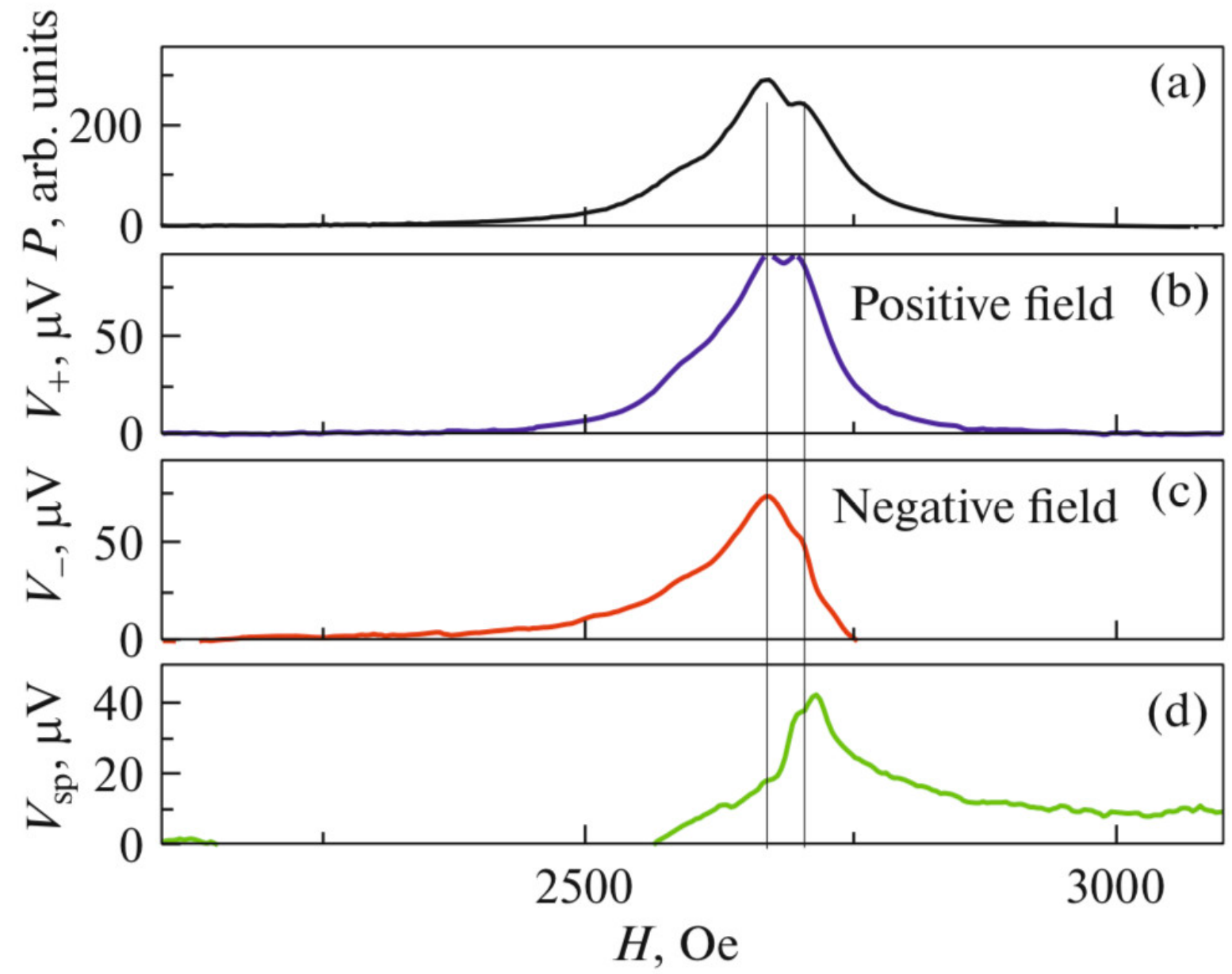

Fig. 7. (a) FMR spectrum of the heterostructure measured in the reflection mode upon the microwave field modulation, (b, c) voltages $V_{+}$and $V_{-}$on the TCFC film in the oppositely directed fields, and (d) film voltage $V_{\text {sp }}$ induced by the spin current flowing through the interface deter- mined by subtracting two voltages for the oppositely directed fields. 

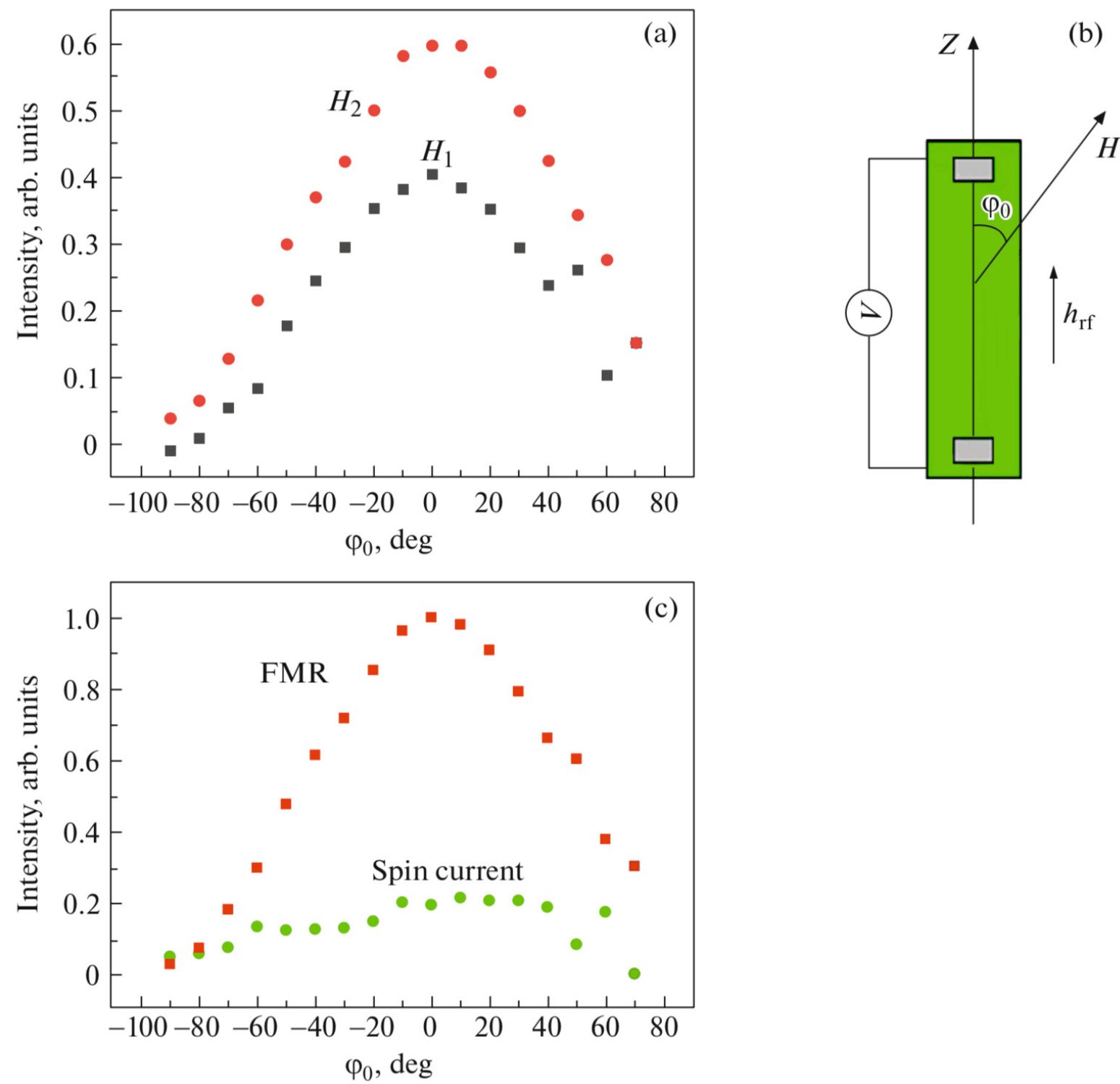

Fig. 8. (a) Angular dependence of the contact voltage line intensity. Squares and circles correspond to two opposite field direc- tions. (b) Angular dependences of the voltage on the TCFC film obtained in the FMR mode (squares) and TCFC film voltage induced by the spin current flow (circles). 